\title{
Gebete in Worten und Taten
}

\section{Das Beispiel der apokryphen Akten des Paulus und der Thekla}

Veronika Niederhofer

Das Thema Gebet als Zeugnis der Herausbildung christlicher Lebensformen und der Ausgestaltung liturgischer Vollzüge ist ein spannendes und aktuelles Forschungsgebiet. ${ }^{1}$ Neben einer übersichtlichen Darstellung zur Forschungsgeschichte des Gebetes im Neuen Testament zeigt zum Beispiel Karl-Heinrich Ostmeyer auf, dass Gebet im neutestamentlichen Verständnis als Kommunikationsgeschehen zu verstehen ist, dessen Besonderheit vor allem an der Rolle Christi festzumachen ist: Gelingendes Gebet ist mit Christus verbunden. Den Schwerpunkt der Analyse neben dem Inhalt des Gebetes vor allem auf die Art des Betens zu legen, ist auch im Hinblick auf "spätere" Texte interessant. Die Schilderungen der Apokryphen Apostelakten beispielsweise zeigen, wie "Christentum» auf neue und andere Weise zugänglich wird und welche bedeutende Rolle dabei auch der Verkündigung mit Wort und Tat zukommt.

Besonders in den Blick rückten in den vergangenen Jahren die apokryphen Akten des Paulus, eine Apostelgeschichte des 2. Jahrhunderts. Als narratives Zeugnis des Eindringens des Christentums in weitere Schichten der hellenistisch-römischen Gesellschaft sind sie auch Mittel der Weiterverbreitung christlichen Glaubens in erzählerischer Form.

Der vorliegende Beitrag konzentriert sich innerhalb der Paulusakten auf die Erzählung um Thekla und stellt deren und Paulus' Formen des Glaubens und Betens dar.

Gerade der narrative Stil kann dabei die neutestamentliche Darstellung ergänzen: anhand verschiedener Erzählelemente können -

\footnotetext{
1 Eine umfassende Studie zum Thema Gebet im Neuen Testament gibt Karl-Heinrich Ostmeyer, Kommunikation mit Gott und Christus. Sprache und Theologie des Gebetes im Neuen Testament (WUNT 197), Tübingen 2006, wie auch Hermut Löhr, Studien zum frühchristlichen und frühjüdischen Gebet. Untersuchungen zu $1 \mathrm{Clem}$ 59-61 in seinem literarischen, historischen und theologischen Kontext (WUNT 160), Tübingen 2003. Der Sammelband von Hans Klein/Vasile Mihoc/Karl-Wilhelm Niebuhr (Hg.), Das Gebet im Neuen Testament (WUNT 249), Tübingen 2009, bietet Studien auch im Bereich Frühjudentum und frühes Christentum.
} 
neben der gesprochenen Form des Betens - weitere Möglichkeiten des Gebetes besonders ausdrucksstark aufgezeigt werden.

\section{Die Akten des Paulus und der Thekla}

Die Überlieferungslage der apokryphen Paulusakten ${ }^{2}$ ist wegen der unterschiedlichen Textzeugen schwierig. Zwei bedeutende Editionen des 20. Jahrhunderts ${ }^{3}$ lassen die bis dahin bekannten drei größeren Textabschnitte - Theklaakten (A. Paul. et Thecl.), den Briefwechsel mit den Korinthern (3 Kor) und ein Martyriumsbericht (Mart. Paul.) - als Bestandteile eines großen Gesamtwerkes erkennen, das nicht vollständig erhalten ist, aber anhand der Aufenthaltsorte ${ }^{4}$ des Apostels rekonstruiert werden kann. ${ }^{5}$

Anhaltspunkte zur Datierung liefert Tertullian, der um die Wende des 2. zum 3. Jahrhundert in seiner Schrift De baptismo 17,5 auf die Paulusakten eines Presbyters in Kleinasien Bezug nimmt. ${ }^{6}$ Damit ist vielleicht auch ein Hinweis zu Verfasserschaft und Lokalisierung der Texte gegeben, möglicherweise kann man von einem gebildeten Presbyter Kleinasiens als Verfasser ausgehen. ${ }^{7}$

2 Die kürzlich erschienene Arbeit von G. Snyder weist darauf hin, den Ausdruck "Paulusakten" und das damit verbundene mögliche Missverstehen mitzubedenken. Die ActPl seien eine Zusammenstellung verschiedener Paulustraditionen, die nebeneinander im Umlauf waren und durch "composition, reception and development of the traditions" als Acts of Paul bewahrt/erhalten sind. Glenn E. Snyder, Acts of Paul. The Formation of a Pauline Corpus (WUNT 2/352), Tübingen 2013, Introduction.

3 Carl Schmidt ediert einen koptischen Papyrus des 6. Jahrhunderts (PHeid) und einen griechischen, entstanden um 300 (PHamb), die zusammen mit weiteren kleinen Papyrusfunden einen Einblick in die wesentlichen Bestandteile der A. Paul. geben.

4 Folgende Stationen des Weges werden erwähnt: Damaskus - Jerusalem - Antiochien - Ikonium - Antiochien - Myra - Sidon - Tyrus - Smyrna - Ephesus - Philippi Korinth - Italien - Rom.

5 Die Angaben berufen sich auf Wilhelm Schneemelcher (Hg.), Neutestamentliche Apokryphen in deutscher Übersetzung. Bd. 2: Apostolisches Apokalypsen und Verwandtes, Tübingen 1989 (= Tübingen ${ }^{6} 1997$ ), 197f, Martin Ebner/Markus Lau, Überlieferung, Gliederung und Komposition, in: Martin Ebner (Hg.), Aus Liebe zu Paulus? Die Akte Thekla neu aufgerollt (SBS 206), Stuttgart (2005), 1-11 und HansJosef Klauck, Apokryphe Apostelakten. Eine Einführung, Stuttgart 2005.

${ }_{6}$ Die Frage nach der genaueren Datierung der Akten des Paulus und der Thekla als eine Quelle der Paulusakten ist jedoch deutlich komplexer. Dazu weiterfuihrend J.N. Bremmer, The Apocryphal Acts: Authors, Place, Time and Readership, in: The Apocryphal Acts of Thomas (hg. v. J.N. Bremmer/I. Czachesz), Leuven (2001), 149-170.

7 Vgl. Klauck, Apokryphe Apostelakten, 64. 
Die Bekehrungserzählung einer jungen Frau namens Thekla ist ein Erzählkomplex innerhalb der Paulusakten. Der größere Kontext kann in zwei Einheiten gegliedert werden, die jeweils ein Martyrium der Thekla schildern.

\section{Das erste Martyrium der Thekla (1-22)}

Paulus flieht aus Antiochien Richtung Ikonium. Der Text erzählt zuerst von der Begegnung mit dem Haus des Onesiphorus und schließt daran die Begebenheiten um Theklas erstes Martyrium an. Thekla lauscht den Worten des Apostels, der im Haus nebenan bei der Familie des Onesiphorus einkehrt und dort das Wort Gottes von der Enthaltsamkeit und der Auferstehung verkündet. In diesem Zusammenhang wird Thekla vorgestellt. Die "Jungfrau" ( $\pi \alpha \rho \theta \varepsilon \dot{v}$ o $\$ 7$ ) ist Tochter der Theoklina und mit Thamyris verlobt. Sie verweilt am Fenster, um die Worte des Paulus zu hören.

"Und während Paulus so sprach in der Gemeinde im Hause des Onesiphorus, sa $\beta$ eine Jungfrau (namens) Thekla - ihre Mutter war Theoklina -, die mit einem Mann (namens) Thamyris verlobt war, an einem dem Haus benachbarten Fenster und hörte Nacht und Tag das Wort vom jungfräulichen Leben, wie es von Paulus verkündet wurde. Und sie neigte sich nicht vom Fenster fort, sondern drängte sich im Glauben und über alle Maßen voller Freude herzu. Da sie aber auch noch viele Frauen und Jungfrauen zu Paulus hineingehen sah, hatte sie das Verlangen, auch sie möchte gewürdigt werden, vor dem Angesicht des Paulus zu stehen und das Wort Christi zu hören. Denn sie hatte Paulus von Angesicht noch nicht gesehen, sondern hörte nur sein Wort." (\$7)

Thekla verharrt am Fenster, ist zunehmend begeistert von den Worten des Apostels und drängt darauf, ihn nicht nur zu hören sondern auch zu sehen. Sie beobachtet zahlreiche junge Frauen, die in das Nachbarhaus eintreten und wünscht, auch unter ihnen zu sein. Von "Glauben" gedrängt und "über alle Maßen voller Freude» ( $\dot{\alpha} \lambda \lambda \grave{\alpha} \tau \tilde{n}$

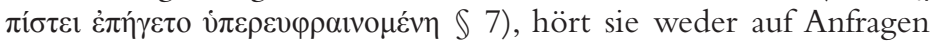
ihres Verlobten noch auf Ermahnungen ihrer Mutter.Thekla ist auch zunehmend überzeugt von der Lehre des Apostels. Ihr Verlobter erkennt dies, und mit Hilfe der Reisebegleiter des Paulus wiegeln sie die Menge gegen den Apostel auf und bringen ihn gemeinsam vor 
den Statthalter. Thekla folgt Paulus sogar ins Gefängnis, um seine Worte dort aufnehmen zu können.

"[...] und sie setzte sich ihm zu Füßen und hörte [ihn] die großen Taten Gottes [verkünden]. Und Paulus fürchtete nichts, sondern wandelte voller Zuversicht zu Gott. Und ihr Glaube nahm zu, und sie küsste auch seine Fesseln.«(\$ 18)

Ihr Verlobter sieht sie mit eigenen Augen vor Paulus im Gefängnis sitzen und veranlasst, dass der Statthalter ihn und auch Thekla vor den Richterstuhl führen lässt. Thekla wird zurVerbrennung auf den Scheiterhaufen verurteilt und wird auf wundersame Weise durch einsetzenden Niederschlag aus dem Feuer gerettet.

\section{Theklas beredtes Schweigen}

Ein Blick auf die Wortelemente zeigt, dass die Erzählung bis hierher nur Worte des Paulus direkt oder indirekt wiedergibt.

Folgendes kennzeichnet die Paulusreden: er spricht im Kontext einer Zusammenkunft im Haus des Onesiphorus "das Wort Gottes von der Enthaltsamkeit und der Auferstehung (\$ 5). Unter den Versammelten herrscht "große Freude«, man bricht Brot und beugt die Knie. Die Worte des Paulus haben den Tenor einer Predigt und führen die Hörerschaft in die Lehre ein. Ihm gehört ungeteilte Aufmerksamkeit, eine Vielzahl an jungen Frauen ist bei ihm ( $\mathbb{9} 9$ ).

Thekla dagegen wird schweigend beschrieben. Ihr Gebet zeigt sich aber in der ersten Erzählhälfte auf andere Art und Weise. Nicht ihre Worte, sondern ihre Taten können nahezu als "wortloses Gebet « angesehen werden. IhrVerhalten, äußerlich wie innerlich, sprechen für sie.

In ihrem Haus verweilt sie Tag und Nacht am Fenster. Glaube und Freude bewegen sie dazu, Paulus nicht nur zu hören, sondern auch zu sehen. (\$ 7) Sie ist gefangen von den Worten des Paulus, spricht nicht mehr, blickt nach unten. Auf nahe Angehörige reagiert sie nicht, diese bezeichnen sie als verstört und weinen über ihrVerhalten (\ 9-10). Ihre Mutter vergleicht sie mit einer Spinne, die am Fenster klebt (\$ 9$)$. 
"Denn alle Frauen und jungen Leute gehen zu ihm hinein und lassen sich von ihm belehren. `Man muss`, sagt er, >einen einzigen Gott allein fürchten und enthaltsam leben aber auch noch meine Tochter, die wie eine Spinne am Fenster klebt, durch seine Worte [bewegt und] von einer nie gekannten Begierde und unheimlichen Leidenschaft ergriffen. Ist doch das Mädchen ganz auf seine Rede ausgerichtet und läßt sich davon gefangen nehmen.« (\$ 9)

Thekla ist sogar bereit, Paulus im Gefängnis aufzusuchen. Um sich Zugang zu ihm zu verschaffen, gibt sie wertvolle Gegenstände an die Gefängniswärter $\mathrm{ab}(\mathbb{S} 18)$. Auch im Gefängnis versucht sie ihrem Lehrer besonders nah zu sein, indem sie sich ihm zu Füßen sitzt und sogar seine Fesseln küsst ( $(18)$. Sie solidarisiert sich soweit mit ihm, dass sie sogar als "mitgefesselt durch ihre Liebe« beschrieben wird. Auch nachdem Paulus aus dem Gefängnis zum Verhör abgeführt ist, bleibt sie am selben Ort.

"Thekla aber wälzte sich auf der Stelle, wo Paulus lehrte, als er im Gefängnis saß« (\$ 20)

Selbst auf ihrem Weg zum Verhör erfüllen sie »Freude« und »Frohlocken« (\$ 20), sie ist ganz auf Paulus fixiert und sucht auch nach seiner Abführung nach ihm. Soweit sogar geht ihre Zuneigung, dass sie ihn über den Zuschauern am Himmel sieht, in Gestalt des Herrn (§ 21$){ }^{8}$

»Thekla aber suchte, wie ein Lamm in der Wüste nach dem Hirten umherschaut, nach Paulus. Und als sie über die Volksmenge hinwegblickte, sah sie den Herrn in der Gestalt des Paulus sitzen und sagte: "Als ob ich nicht standhaft wäre, ist Paulus gekommen, um nach mir zu sehen." Und sie schaute auf ihn unverwandt; er aber entschwand in den Himmel.« (\$ 21)

Den Scheiterhaufen besteigt sie auf ganz besondere Weise, indem

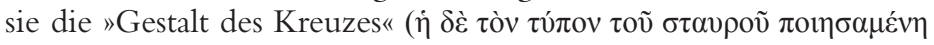
$\left.\dot{\varepsilon} \pi \dot{\varepsilon} \beta \eta \tau \tilde{\omega} v \xi \hat{v} \lambda \omega v \cdot \int 22\right)$ dabei macht.

Thekla schweigt also im bisherigen Erzählverlauf, ihre Äußerung in $\int 21$ ist eher als Selbstgespräch zu verstehen. Weder ihrer Familie noch beim Verhör gibt sie Antwort, doch auch ihr Schweigen spricht.

\footnotetext{
8 Dies sind die ersten Worte im Erzählverlauf, die Thekla in den Mund gelegt werden.
} 


\section{Die Gebete von Paulus und Thekla nach der Rettung (23-25)}

Nach der Rettung macht sich Thekla auf die Suche nach Paulus, trifft auf ein Kind des Onesiphorus und lässt sich zu Paulus führen, der bereits sechs Tage fastet, seufzt und betet. Thekla tritt hinter ihn und es folgt ein Wechsel an Gebeten:Thekla vernimmt das Bittgebet des Paulus, fügt dem ein Dankgebet hinzu, worauf er in einen weiteren Dank einstimmt $(\$ 24)$.

»Vater Christi, möge das Feuer Thekla nicht anrühren, sondern stehe du ihr bei, denn sie ist dein!«

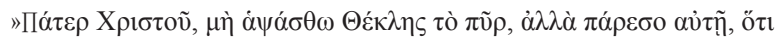

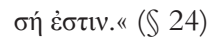

Zuerst also bittet Paulus um die Rettung Theklas. Der Aufbau der Worte des Paulus, eine Anrede und eine entfaltete Bitte, folgen dem Grundschema eines Gebetes. Er spricht Gott an, er nennt ihn Vater (Пó $\tau \varepsilon \rho)$ und bittet, das Feuer möge Thekla nicht anrühren. Gott soll

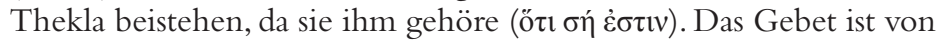
Fasten und Seufzen begleitet ( $\mathbb{S} 23)$. Seine Haltung, gebeugte Knie ${ }^{9}$, entspricht der Art des Gebetes, der Bitte. Daran schließt sich das Gebet der Thekla. Es klingt wie eine Antwort auf die Bitte des Paulus:

"Vater, der du den Himmel und die Erde gemacht hast, du, der Vater deines geliebten Sohnes [Jesus Christus], ich preise dich, dass du mich aus dem Feuer gerettet hast, damit ich Paulus sehe!«

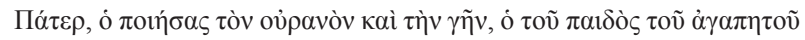

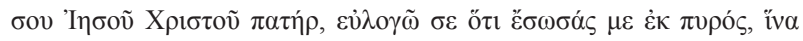

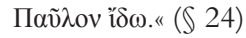

In der Erzählung ist es das erste Gebet, das Thekla spricht. Der Aufbau der beiden Gebete ist der Form wie auch dem Inhalt nach ähnlich:Thekla verwendet dieselbe Anrede wie Paulus, fügt dem Inhalt gemäß dieselben Glaubensaussagen an und spricht dann einen Lob-

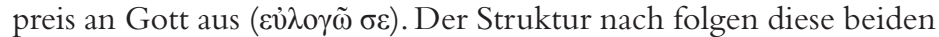
Elemente des Gebetes auch dem des Paulus. Er schließt der Anrede eine Bitte an, Thekla einen Lobpreis. Inhaltlich sprechen beide von der Thematik Feuer - Paulus bittet um die Bewahrung der Thekla

\footnotetext{
9 Auch im Epheserbrief 3,14-16 wird das Bittgebet mit der Gebetshaltung des Kniebeugens verbunden ("Daher beuge ich meine Knie vor dem Vater, nach dessen Namen jedes Geschlecht im Himmel und auf der Erde benannt wird, und bitte ... «).
} 
davor, diese dankt für ihre Rettung. ${ }^{10}$ Abschließend folgt, ebenfalls analog gestaltet zum Gebet des Paulus, eine Erklärung der Bitte/ des Lobpreises mithilfe eines Adverbialsatzes. Paulus begründet seine Bitte mit einem Kausalsatz (ö $\tau$ ), Thekla mit einem Finalsatz (iv $\alpha$ ).

Diesem Gebet der Thekla folgt ein weiteres des Paulus. Er sieht sie lebend, ändert daraufhin seine Gebetshaltung und steht auf und spricht:

"Gott, du Herzenskundiger,Vater unseres Herrn Jesu Christi, ich preise dich, dass du das, worum ich [dich] bat, so schnell [getan hast] und mich erhört hast."

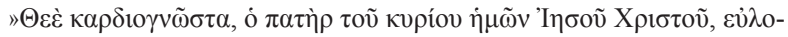

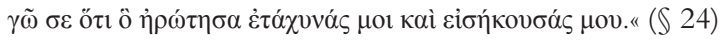

Diese Worte wiederum erscheinen als Antwort auf das Gebet der Thekla. Wie die beiden vorausgehenden Gebete beginnt auch dieses mit einer Anrede an Gott, der nun auch als $\Theta \varepsilon \dot{\varepsilon}$, Gott, bezeichnet wird. Es folgen zwei Ausführungen zu diesem Gott. Er wird als

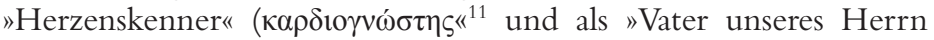

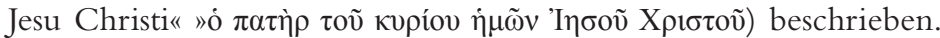
Im Gebet folgt nach einer ausgestalteten Anrede der Lobpreis, der

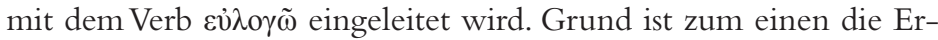
hörung der Bitte, zum anderen die schnelle Erfüllung dieser durch Gott.

\footnotetext{
${ }^{10}$ Der Unterschied der Gebete von Paulus und Thekla besteht auch darin, dass Paulus um Gottes Beistand furr Thekla bittet, also die Beziehung Gott - Thekla betont, Thekla dagegen im Gebet eine Beziehung thematisiert, die sie und Paulus betrifft. Sie dankt Gott dafür, dass sie Paulus sehen kann.

11 "Herzenskenner" ist eine Anrede, die in Apg 1,24 und 15,8 verwendet wird, ansonsten aber nicht häufig belegt ist, vgl. Klauck, Apokryphe Apostelakten, 86.
} 
Vergleich der Gebete von Paulus und Thekla (24):

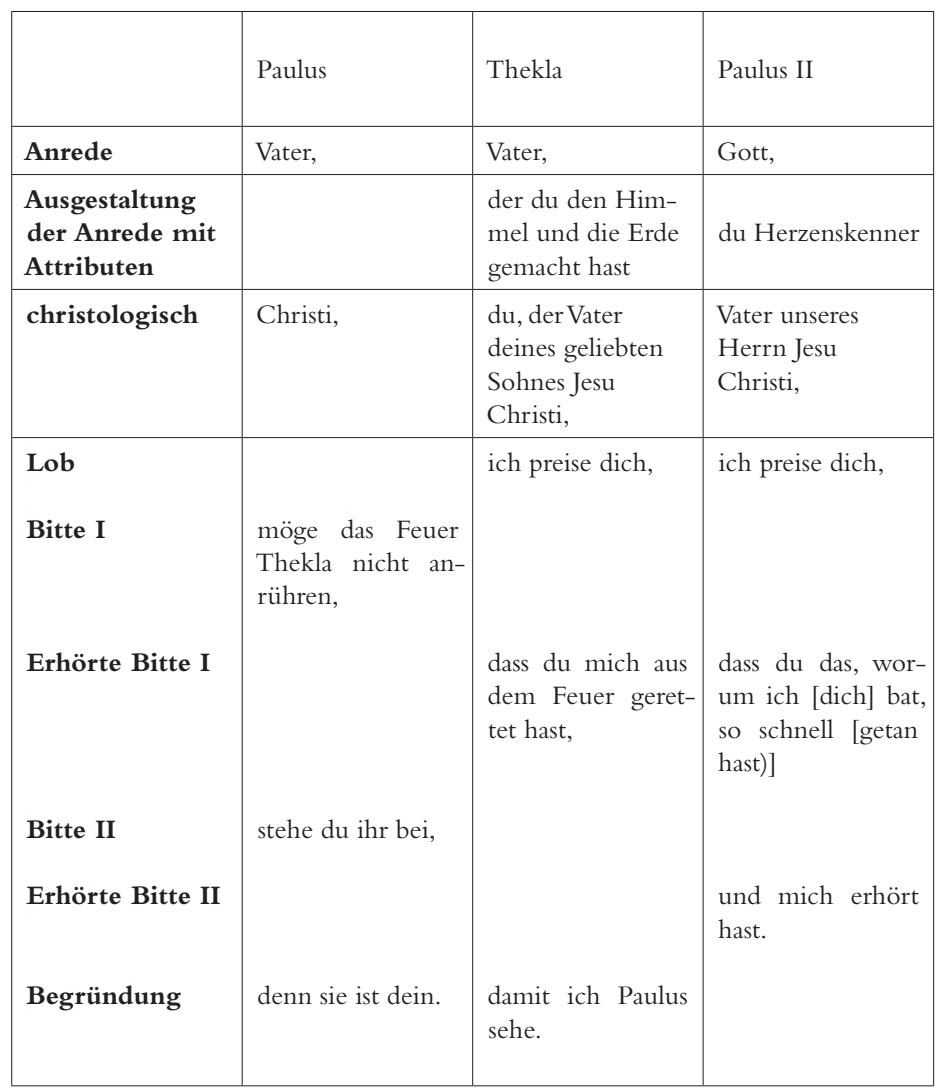

Nach dem Zusammentreffen und den Gebeten folgt ein weiterer Schritt der Thekla: sie möchte Paulus nachfolgen und bittet ihn um "das Siegel in Christo" (25), die Taufe. Ihre Verlobung ist damit von ihrer Seite her hinfällig. 


\section{Gebet in Wort und Tat}

Thekla spricht weder mit Familienangehörigen, wie mit ihrer Mutter oder ihrem Verlobten Thamyris, noch gibt sie Autoritäten Antwort. Sie verweigert dem Prokonsul die Aussage, indem sie auf seine Frage hin schweigt. Selbst in schlimmen Situationen, vor und während derVerbrennung auf dem Scheiterhaufen, spricht sie keine Bitt- oder Klagegebete.

Thekla kommt zwar so nicht aktiv zu Wort, doch spricht sie zum Leser durch ihr Schweigen auf direkt an sie gerichtete Fragen. So sehr gefestigt und treu ist sie im neuen Glauben bereits, der allein durch das Hören verursacht wurde.

Erst nach derVerurteilung erhebt sie zum ersten Mal die Stimme, wenn sich die Menge bereits ins Theater begibt. Auf der Suche nach Paulus äußert sie "Als ob ich nicht standhaft wäre, ist Paulus gekommen, um nach mir zu sehen« (' $\Omega \varsigma \dot{\alpha} v v \pi$ o $\mu$ ov

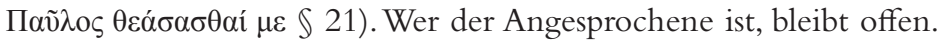
Die Aussage erscheint eher wie ein Selbstzuspruch, ein Gedanke, der laut geäußert wird.

Nach ihrer Rettung spricht Thekla mit einem Kind aus dem Haus des Onesiphorus und mit Paulus lässt sie einen Dialog zu. Zugespitzt kann man auch formulieren, dass Thekla nur mit bereits glaubenden Personen spricht, mit denen, die auch Paulus nachfolgen und auch zu seinem Gott gehören.

So wie ihr ganzes Verhalten auf die Person des Paulus ausgerichtet ist, spiegeln auch ihre Worte diese wieder. Sie glaubt, Paulus zu sehen, der zu ihr in der Not gekommen ist $(\$ 21)$ und ist nach der Rettung auf der Suche nach ihm (\$23).

Thekla bewahrt ihre Fassung, aber verändert zugleich ihr Verhalten. Ihr genügt es nicht mehr, Paulus nur vom Nachbarhaus aus zu hören: sie will ihn sehen, sie verfolgt ihn, berührt ihn und sucht ihn nach der Vernehmung solange, bis sie eine Gestalt am Himmel erblickt, die sein Aussehen hat. ${ }^{12}$ Sie sucht ihn nach ihrer Rettung und spricht auf sein Bittgebet hin selber ein Dankgebet. Nachfolge und die Bitte um das Siegel kennzeichnen ihrVerhalten weiter.

Unterschiedliche Gebetshaltungen werden genannt:

Die Gebetshaltung des Apostels wie auch der anderen Versammelten ist beim Verkünden gebeugt $(\mathbb{S} 5 ; 24)$. Nach dem Gebet in

\footnotetext{
12 An dieser Stelle sind erotische Untertöne, die in manchen Aussagen und Erzählpassagen anklingen, unüberhörbar.Vgl. hierzu z.B. J.W. Barrier, The Acts of Paul and Thecla. A Critical Introduction and Commentary (WUNT 2/270), Tübingen 2009, 86.
} 
der Grabkammer wird explizit gesagt, dass sich Paulus erhebt und dann Thekla erblickt, worauf er sein Gebet anscheinend im Stehen fortführt (S 24). Sein Klagegebet erfolgt im Knien, der Dank im Stehen. Bestimmte Haltungen korrespondieren einem bestimmten Thema - Körpersprache dient hier als Ausdruck und Verstärkung der Gebetsworte.

\section{Das Zweite Martyrium der Thekla (26-43)}

Nach ihrer Rettung beginnt die Erzählung sogleich mit der Handlung, die zum zweiten Martyrium der Thekla führt. Der Erzählverlauf zeigt, dass mehrere Aussagen der Thekla aufgrund der Struktur wiederum als Gebete charakterisiert werden können.

Thekla begleitet Paulus nach Ikonium. Dort widersagt sie dem Werben des mächtigen Alexander, worauf sie wiederum verurteilt wird, diesmal zum Tierkampf. Eine reiche Frau namens Tryphäna nimmt sie vorübergehend bei sich auf. Diese bittet Thekla, für ihre eben verstorbene Tochter zu beten ( $(28)$.

»Sie aber erhob, ohne zu zögern, ihre Stimme und sprach: >Mein Gott im Himmel, Sohn des Höchsten, verleihe ihr nach ihrem Willen, dass ihre Tochter Falconilla leben möge in Ewigkeit!« $(\mathbb{S} 29)$

Thekla wird zum einen aufgefordert zum Gebet, zum anderen wird ihr und ihrem Gebet eine besondere Wirkung zugetraut: ewiges Leben bei der Verstorbenen. Am Tag des Tierkampfes wird Thekla von Soldaten des Alexander, dem Veranstalter, abgeholt.Tryphäna spricht ihr ihren Beistand aus, worauf Thekla sich voller Tränen und Seufzen an ihren Herrn wendet. Das Gebet, eine Bitte, enthält gattungsspezifische Merkmale. Sie spricht ihn als Kúpıє ó $\theta$ cós an. Diese Anrede wird weiter ausgestaltet. Thekla beschreibt ihren Gott als

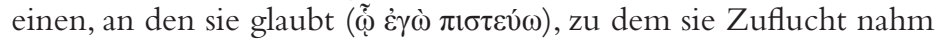
und der sie gerettet hat. Dass sie glaubt, und warum sie glaubt, wird in einem Zug erwähnt. In ihrer Situation, die wiederum ausweglos erscheint, kann sie bereits sagen, dass sie an diesen Gott glaubt. Aufgrund ihrer Vorgeschichte drückt sie damit die Hoffnung, aber auch die Zuversicht aus, dass sich Gott wieder als helfend erweisen wird. Was sie von Gott erwartet, formuliert sie in der folgenden Bitte: er möge Tryphäna belohnen. Dem schließt sich eine zweifache Begründung an: Tryphäna hatte zum einen mit Thekla Mitleid, zum anderen hat sie sie dadurch "rein bewahrt«. 
"Herr Gott, an den ich glaube, zu dem ich meine Zuflucht genommen habe, der mich aus dem Feuer errettet hat, belohne Tryphäna, die mit deiner Dienerin Mitleid hatte, so dass sie mich rein bewahrt hat." $(\mathbb{S} 31)$

Auch während Tiere in die Arena geführt werden, steht Thekla still und betet. Anschließend wendet sie sich und sieht eine Grube. Dieser Anblick weckt in ihr den Gedanken an die Taufe. Die Worte des Gebets sind nicht bekannt, wohl ist eher an ein Fürbittgebet um Hilfe und Rettung in ihrer Notsituation zu denken. ${ }^{13}$

»Da ließen sie viele Tiere herein, während sie dastand und die Hände ausgebreitet hatte und betete. Als sie aber ihr Gebet beendet hatte, wandte sie sich um und sah eine große Grube voll Wasser und sprach: Jetzt ist der Zeitpunkt gekommen, mich zu waschen.< Und sie stürzte sich selbst hinein mit den Worten: \Im Namen Jesu Christi taufe ich mich am letzten Tag!« (\$ 34)

Im weiteren Erzählverlauf folgen den Bitt- und Dankgebeten Aussagen Theklas und weiterer Figuren, die in ihrem Charakter eher Bekenntnissen entsprechen, die sie bzw. andere von Gott ablegen.

Der Statthalter lässt Thekla aus dem Tierkampf holen, nachdem der mächtige Alexander seine Bedenken bzgl. der Hinrichtung Theklas geäußert hatte. Er fragt sie, wer sie sei, und, weil ihn verwundere, dass sogar Tiere vor ihr Halt machen, was es mit ihr auf sich habe. Als Antwort gibt Thekla eine umfassende Auskunft. Die erste Frage beantwortet sie knapp, sie versteht sich als »eine Dienerin des le-

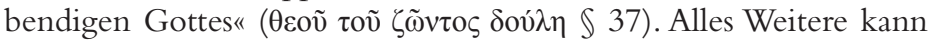
als Bekenntnis gesehen werden, das umfassende Auskunft über den Gott, den sie verehrt, gibt. Sie begründet, weshalb sie die Tiere unberührt lassen folgendermaßen: ihr Glaube an Gottes Sohn, der »Ziel der Rettung und die Grundlage unsterblichen Lebens" ( $\sigma \omega \tau \eta \rho i ́ \alpha$

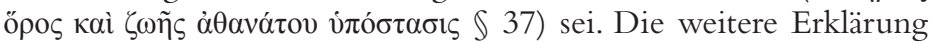
verallgemeinert die Aussage: der Glaube an den Sohn, Christus, ist die Voraussetzung für Rettung auf Erden und für Rettung auf Dauer.

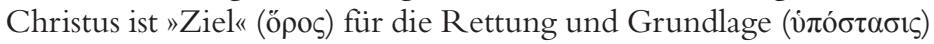
der Unsterblichkeit.

Daran schließen sich Beispiele, die den Sohn in seiner Funktion als Retter ${ }^{14}$ beschreiben. Drei Lebenslagen - Geplagtheit durch den

13 Das Gebet ist also nicht als Vorbereitungsgebet zur Taufe zu verstehen. Erst den

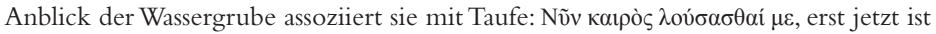
der Kairos, der richtige Zeitpunkt. Sie möchte sich "waschen«.

${ }^{14}$ Vgl. A. Paul. 2,29: Paulus spricht zu Artemilla und spricht die Retterfunktion Got-

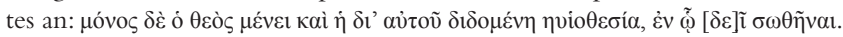


Sturm, Bedrängnis und Verzweiflung - werden genannt, in denen Rettung in Form von Zuflucht, Erquickung und Schutz gewährt wird. Das Bekenntnis endet mit einer zusammenfassenden Aussage, die den Glauben an Jesus Christus in das Zentrum stellt. Um Leben anstatt Tod in Ewigkeit zu erlangen, ist ein "Glauben an ihn" ( $\pi 1 \sigma \tau \varepsilon v ́-$

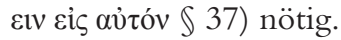

Nach der Freilassung durch den Statthalter äußern auch die Frauen der Stadt, die das Geschehen beobachtet haben, große Worte:

"Die Frauen aber schrien alle mit lauter Stimme und lobten Gott wie aus einem Munde und sprachen: ' Einer ist Gott, der Thekla gerettet hat $\iota$ so dass von dem Schreien die ganze Stadt erbebte." (\$ 38)

Tryphäna vernimmt die Worte des Statthalters und trifft eine allgemeine Glaubensaussage: "Jetzt glaube ich, dass die Toten erweckt

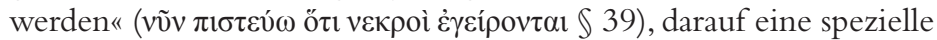

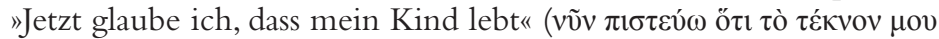
$\zeta \tilde{n}$ \$ 39). Aus dem sichtbaren Werk der Rettung Theklas durch Gott folgert sie, dass Gott auch auf die Bitten einer solchen Person hören wird. ${ }^{15}$ Sie glaubt daher daran, dass die Bitte Theklas, die ewiges Leben für ihre Tochter erbeteten hat, erhört wird.

Nach ihrer Rettung und Erholung bei Tryphäna sucht und findet Thekla Paulus. Sie berichtet von den Geschehnissen um ihre Rettung und von ihrer Taufe.

"Sie selbst aber zog nach Ikonium und trat in das Haus des Onesiphorus und warf sich auf den Boden, wo Paulus gesessen und die Worte Gottes gelehrt hatte, und sie weinte und sprach: >Mein Gott und [Gott] dieses Hauses, wo mir das Licht leuchtete, Christus Jesus, der Sohn des Gottes, mein Helfer im Gefängnis, Helfer vor Statthaltern, Helfer im Feuer, Helfer unter den Tieren, du selbst bist Gott und dir sei Ehre in die Äonen/Ewigkeiten, Amen.« ( $(42)$

Thekla führt den Auftrag des Paulus aus und verkündet das »Wort Gottes" ( $(41)$ im Haus des Onesiphorus. Der Struktur nach gleichen ihre Worte wiederum einem Gebet mit folgendem Aufbau:

Sie spricht Gott an, und gibt ihm zwei Attribute. Er ist ihr Gott und der "ihres Hauses". Daran schließen sich Worte, die das "Haus" näher beschreiben. Die Ereignisse »dort« (öлov) haben Thekla Klar-

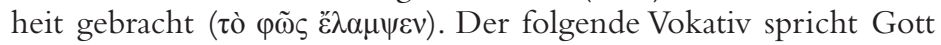

${ }^{15}$ Aussagen derart könnten zu späterer Zeit zu dem Pilgerkult um Thekla geführt haben, wie sie Egeria beschreibt (Eg. Itin. 23,5). 


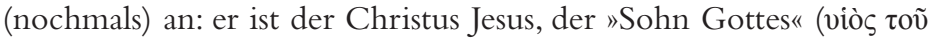
$\theta \varepsilon o v)$, der ihr in vielen Situationen geholfen hat. Ihn hat sie um ewiges Leben für Falconilla angerufen (\$ 29), und aufgrund der eigenen erfahrenen Hilfe kann sie ihn als Gott bezeichnen; so spricht sie ihm "Ehre in Ewigkeit« zu. Das folgende "Amen« schließt das Gebet der Thekla ab. Auch an dieser Stelle erfolgt wieder der Hinweis auf eine sinnliche Komponente des Glaubens: Thekla geht nicht nur nach Ikonium, sondern auch in das Haus des Onesiphorus und sogar an die Stelle im Haus, von der aus Paulus gesprochen hat. Gebet wird mit Nachfolge und Glaube in Verbindung gebracht und steht zudem wieder im Kontext von gewissen rituellen Handlungen. ${ }^{16}$

In Ikonium trifft Thekla dann auf ihre Mutter, vor der sie nochmals Zeugnis von Gott ablegt:

»Theoklina, meine Mutter, kannst du glauben, daß ein Herr im Himmel lebt? Denn ob du nach Geld und Gut verlangst, der Herr wird es dir durch mich geben, oder nach deinem Kinde, siehe, ich stehe an deiner Seite.«( (\$ 43)

\section{Theklas Verhalten: Paulusverehrerin, Lehrerin und Pilgerin}

Apokryphe Apostelakten versuchen, neue soziale Schichten zu erschließen. Dies kann z.B. durch die Aufnahme neuer literarischer Formen (wie etwa Roman) oder durch Einbeziehung von ’höhergestellten Personen in die Erzählung, etwa einen Apostel, geschehen. Als »neue Gründungserzählungen« schließen sie an apostolische Zeiten an, füllen auf narrative Weise zugleich scheinbare Erzähllücken der neutestamentlichen Texte, und schildern neue Ereignisse, die auch das steigernde Interesse an hagiographischen Elementen zum Ausdruck bringen. In den Akten des Paulus und der Thekla rückt letztere als vorbildliche Zeugin des Glaubens in den Mittelpunkt des Geschehens. Thekla ist zunehmend von Paulus begeistert, möchte immer mehr von seiner Lehre erfahren und auch danach leben. Sie handelt konsequent und verändert ihr bisheriges Leben.

Ihr ganzes Verhalten richtet sie auf Paulus aus. Ihre innere Einstellung und ihr Tun sind bestimmt von seiner Person. Ihre Verehrung und die damit verbundenen Verhaltensweisen entsprechen denen

${ }^{16}$ Naheliegend wäre auch der Gedanke, dass das Haus, in dem Paulus gesprochen hat, bereits als "heilige" Stätte verstanden und der Umgang dementsprechend achtungsvoll gestaltet wurde. 
von treuen Jüngern und Nachfolgern. In Ansätzen zeigt sie den Lebenswandel einer Pilgerin: sie bricht von zu Hause auf und macht sich auf den Weg dorthin, wo sie Paulus vermutet, um ihrem Vorbild nahe zu sein. Sie besucht Orte, an denen Paulus verweilte und verehrt diese mit bestimmten Gesten und Handlungen.

\section{Theklas Gebet}

\section{Wort und Haltung}

Der kurze Blick in die Erzählungen um Thekla zeigt bereits eine bunte Vielfalt an Ausdrucksformen und Möglichkeiten des Gebetes. Unterschiedliche Gebetshaltungen werden genannt:

Die Haltung des Apostels wie auch der anderen Versammelten ist beim Verkünden gebeugt ( $\$ \$ 5 ; 24)$. In $\$ 24$ wird zwischen Gebetsarten und den jeweiligen Haltungen differenziert: das Klagegebet wird mit gebeugten Knien vollzogen, der Dank im Stehen.

Dass neben verbalen Formen vor allem auch sinnliche und aktionale Elemente eine bedeutende Rolle gewinnen, zeigt vor allem der Blick auf die Protagonistin der Erzählung. Thekla schweigt auf direkt an sie gerichtete Fragen und kommt erst gegen Ende des ersten Erzählabschnittes aktiv zu Wort. Bis dahin spricht sie durch ihr Schweigen und ihr stetes Verhalten nahezu passiv zu ihren Angehörigen und auch zum Leser.

Frommen Pilgern, wie sie aus späterer Zeit bekannt sind, gemäß, spricht sie vor allem im zweiten Erzählteil immer wieder und immer mehr Gebete und erzählt anderen Personen von ihren Erfahrungen. Auch in Form von Bekenntnissen gibt sie weiter, was sie vom Hören und Verweilen bei Paulus von seiner Lehre aufgenommen und verstanden hat.

\section{Leiblichkeit - Gehorsam - Nachfolge}

Thekla könnte so als treue Paulusverehrerin bezeichnet werden, die ihr Verhalten dem von Pilgern späterer Zeit angleicht. Sie gibt sich durch ihr Tun und durch ihr "Gebet" in allen vorgestellten Variationen - verbal, nonverbal, aktional, sinnlich - als Paulusverehrerin und Nachfolgerin, die noch zu Lebzeiten einen Personenkult vollzieht und auch Riten derVerehrung ihres Vorbildes entwickelt. In $\ 42$ lesen wir, dass sie sich vor ihrer Abreise nach Ikonium noch einmal an 
der Stelle, wo Paulus gesessen hat, auf den Boden wirft und zu Gott und Christus betet. So bewahrt sie den Kontakt zum abwesenden Apostel auf neue Weise, die eher kultische Züge trägt.

\section{Anlehnung an das Neue Testament und Zusatz an Information}

Die Analyse der Gebete zeigt eine zum Teil enge Anknüpfung an das, was wir aus dem Neuen Testament kennen. Neben verbalen Ähnlichkeiten sind auch die Gesten - z.B. erhobene Hände - bereits als begleitende Elemente von Gebeten im neutestamentlichen Kontext erwähnt.

Die Erzählung gibt Informationen, die über das Neue Testament hinaus reichen. Die Figur des Paulus nimmt neue Züge an. Er spielt nur am Rande der Erzählung eine Rolle. Er zieht umher, lehrt und belehrt die Einwohner, und tritt mit zunehmender Präsenz der The$\mathrm{kla}$ immer mehr in den Hintergrund. Zugleich übernimmt diese als seine Schülerin die Rolle der Lehrenden und Zeugnisgebenden.

Am Ende der Akten des Paulus und der Thekla kann nun über Thekla selbst gesagt werden, was im ersten Teil der Erzählung noch für Paulus gilt: ihr Zeugnis in Wort und Tat führt andere zum Glauben. ${ }^{17}$

Die Thekla-Episode zeigt vielfaltige Dimensionen des Gebetes auf. Der narrative Charakter vermittelt den Eindruck, als ob Geschehnisse sehr nah "aus dem Leben" geschildert werden. Dieses wiederum kann als Schlüssel zu einer Frömmigkeit gesehen werden, die mehrdimensional ausgestaltet ist. Dem Aspekt der Leiblichkeit kommt auf diese Weise durch kleine narrative Zwischenbemerkungen eine beachtliche Bedeutung zu.

- Dipl. theol.Veronika Niederhofer ist Wissenschaftliche Mitarbeiterin am Lehrstuhl für Exegese und Hermeneutik des Neuen Testaments an der Fakultät für Katholische Theologie der Universität Regensburg.

\footnotetext{
17 Auch der Hinweis auf Seleukia, wo Thekla entschläft (\$ 43), kann in dieser Linie begriffen werden. Seleukia wird mit dem Grab der Thekla zu einem bereits in der Spätantike wichtigen Pilgerzentrum. Die Pilgerin Egeria schreibt in ihrem Reisebericht, dass an diesem Ort zum Gedenken an Thekla die Akten der Thekla gelesen wurden (Itin.Eg. 23,5, um 400).Vgl. dazu Tobias Nicklas, Beyond "Canon«. Christian Apocrypha and Pilgrimage (Vortrag in London 2014).
} 